\title{
Sociodemographic Predictors of Outcomes in COVID-19: Examining the Impact of Ethnic Disparities in Northern Nevada
}

Daniel Antwi-Amoabeng ${ }^{1}$, Bryce D. Beutler ${ }^{2}$, Munadel Awad ${ }^{1}$, Zahara Kanji ${ }^{1}$, Sumaiya Mahboob ${ }^{1}$, Jasmine Ghuman ${ }^{1}$, Sri Harsha Boppana ${ }^{1}$, Mohammad Sheikh ${ }^{1}$, Mark Bilinyi. Ulanja ${ }^{1}$, Nageshwara Gullapalli ${ }^{1}$

1. Internal Medicine, University of Nevada Reno School of Medicine, Reno, USA 2. Radiology, University of Southern California Keck School of Medicine, Los Angeles, USA

Corresponding author: Bryce D. Beutler, brycebeutler@hotmail.com

\section{Abstract \\ Background}

On March 11, 2020, the World Health Organization declared coronavirus disease-19 (COVID-19) a pandemic. Nearly five million individuals have since been diagnosed with this increasingly common and potentially lethal viral infection. Emerging evidence suggests a disproportionate burden of illness and death among minority communities. We aimed to evaluate the effect of ethnicity on outcomes among patients diagnosed with COVID-19 in Northern Nevada.

\section{Methods}

The electronic health records of 172 patients diagnosed with COVID-19 were obtained from a 946-bed tertiary referral center serving Northern Nevada. Demographic and clinical characteristics were compared by ethnic group (Hispanic versus non-Hispanic). Logistic regression was used to determine predictors of mortality.

\section{Results}

Among 172 patients who were diagnosed with COVID-19 between March 12 and May 8, 2020, 87 (50.6\%) identified as Hispanic and 81 (47.1\%) as non-Hispanic. Hispanic individuals were significantly more likely to be uninsured and to live in low-income communities as compared to their non-Hispanic counterparts $(27.6 \%$ versus $8.2 \%$ and $52.9 \%$ versus $30.6 \%$, respectively). Hispanic patients were also less likely than nonHispanics to have a primary care provider (42.5\% versus $61.2 \%$ ). However, mortality was significantly higher among the non-Hispanic population (15.3\% versus $5.8 \%)$.

\section{Conclusion}

Review began 01/16/2021 Review ended 01/31/2021 Published 02/04/2021

\section{() Copyright 2021}

Antwi-Amoabeng et al. This is an open access article distributed under the terms of the Creative Commons Attribution License CC-BY 4.0., which permits unrestricted use, distribution, and reproduction in any medium, provided the original author and source are credited.
The COVID-19 pandemic has disproportionately affected Hispanic individuals in Northern Nevada, who account for only $25.7 \%$ of the population but over half of the confirmed cases. The underlying causes of ethnic disparities in COVID-19 incidence remain to be established, but further investigation may lead to more effective community- and systems-based interventions.

Categories: Internal Medicine, Infectious Disease, Epidemiology/Public Health

Keywords: coronavirus, covid, covid-19, ethnic disaprities, health policy, racial disparities, sars-cov-2

\section{Introduction}

The coronavirus disease-19 (COVID-19) pandemic has changed the face of healthcare in the United States. This pandemic follows two previous coronaviridae pandemics in the last two decades: severe acute respiratory syndrome (SARS) and Middle East respiratory syndrome (MERS) [1,2]. As of this writing, over 25 million Americans have been diagnosed and nearly 450,000 have died of this increasingly common viral infection [3]. Although primarily considered a pulmonary disease, extrapulmonary manifestations of COVID-19 have been reported, including neuropsychiatric, cardiac, ocular, dermatologic, and gastrointestinal symptoms [4-5].

Emerging evidence suggests a disproportionate burden of illness and death among communities of color [68]. The underlying causes of ethnic disparities in the setting of COVID-19 remain to be established, but it has been postulated that social distancing represents a privilege of the dominant ethnic group; minority individuals are more likely to work essential jobs and live in multigenerational households as compared to their white counterparts $[9,10]$. In addition, distrust of the medical establishment is pervasive among minority communities, and public health messages may not resonate among individuals who have been 
affected by systemic racism [11].

Ethnic disparities in COVID-19 outcomes have been reported by other authors. However, there is a paucity of data pertaining specifically to the Hispanic population. In this study, we aimed to compare COVID-19 incidence and outcomes between the Hispanic and non-Hispanic populations of Northern Nevada.

This article was previously posted to the medRxiv preprint server on May 25, 2020.

\section{Materials And Methods}

\section{Source of data}

We conducted a single-center, retrospective observational study using data extracted from the electronic health records of a 946-bed tertiary referral hospital serving most of Northern Nevada and affiliated satellite hospitals.

\section{Study population}

The study sample included all patients who had a positive nasopharyngeal reverse-transcriptase polymerase chain reaction test for severe acute respiratory syndrome coronavirus 2 (SARS-CoV-2) between March 12 and May 8, $2020(\mathrm{~N}=172)$.

\section{Variables}

The primary outcome of interest was mortality. Secondary outcomes included the following: hospitalization, acute respiratory distress syndrome (ARDS), sepsis, shock, mechanical ventilation, hepatic injury, renal injury, arrhythmia, coagulopathy, and hemorrhage (as defined by the International Classification of Diseases, 10th revision [ICD-10] coding). Additional patient-level data included age, sex, comorbidities, ethnicity (Hispanic or non-Hispanic), income class (low or middle/upper), insurance type (Medicare, Medicaid, private, uninsured, or workers' compensation), and the presence or absence of a primary care provider.

\section{Statistical analyses}

Categorical variables were described using proportions and were compared among ethnic grouping with Fisher's exact test. Continuous variables were not normally distributed and were described as medians and interquartile ranges (IQRs). Sex, age categories, ethnic grouping, presence of diabetes, hypertension, obesity, chronic kidney disease, chronic obstructive pulmonary disease, and intensive care unit stay were used as predictors to fit a logistic regression model for the outcome of death. The Hosmer-Lemeshow test was used to assess the goodness of fit of the model. The variance inflation factor (VIF) was used to detect the presence of multiple collinearity among the model predictors; collinearity was not detected among variables. The Mann-Whitney U test was used to assess the distribution of coagulation profiles among survivors and non-survivors. All tests were performed as two-tailed and statistical significance levels set at a $\mathrm{P}$ value $<.05$ in all applied analyses using Stata, version 16 (StataCorp., College Station, Texas).

\section{Results}

A total of 172 patients tested positive for SARS-CoV-2 between March 12 and May 8, 2020. Men were slightly overrepresented as compared to women (96 [55.8\%] versus 76 [44.2\%], respectively). The median age was 53 years (range: 33.5 - 68 years). A significant majority of patients were under the age of 61 years (113 [65.7\%]). Hospitalization was required for 121 patients (70.3\%); 51 patients (29.7\%) were discharged home and instructed to self-isolate. Among all 121 patients who were hospitalized, 28 (23.1\%) were treated in the intensive care unit and 73 (60.3\%) were successfully discharged. A total of 18 patients (10.5\%) died.

A review of the distribution of cases based on ethnicity revealed that 87 individuals $(50.1 \%)$ identified as Hispanic and 85 (49.4\%) identified as non-Hispanic. Notably, Hispanics account for only $25.7 \%$ of the population of Northern Nevada. Hispanic individuals were significantly younger than their non-Hispanic counterparts, with a mean age of 46.0 versus 55.8 years, respectively. Furthermore, 68 (78.2\%) Hispanic patients were under the age of 61 years whereas only 45 (52.9\%) of non-Hispanic patients were under the age of 61 years (Table 1 ).

\begin{tabular}{|c|c|c|c|}
\hline & \multicolumn{2}{|c|}{ Patients, No (\%) } & \multirow[t]{2}{*}{ p-value } \\
\hline & Non-Hispanic & Hispanic & \\
\hline \multicolumn{4}{|l|}{ Age } \\
\hline Less than 61 years & $45(52.94)$ & 68(78.16) & 0.001 \\
\hline 61 years and above & $40(47.06)$ & 19(21.84) & \\
\hline
\end{tabular}




\section{Cureus}

Sex

46(54.12)

50(57.47)

0.759

Female, $\mathrm{n}=76$

39(45.88)

$37(42.53)$

Income Class

Low income

26(30.59)

46(52.87)

0.003

Middle/Upper income

59(69.41)

41(47.13)

Primary care provider (PCP)

Has a PCP

52(61.18)

37(42.53)

0.015

Does not have PCP

33(38.82)

$50(57.47)$

Insurance Type

Medicare

28(32.94)

9(10.34)

$<0.001$

Medicaid

15(17.65)

15(17.24)

Private

32(37.65)

32(36.78)

Uninsured

$7(8.24)$

24(27.59)

Workman's comp

$3(3.53)$

7(8.05)

Comorbidities

Hypertension

45(52.94)

25(28.74)

0.002

Diabetes

23(27.06)

26(29.89)

0.737

Dyslipidemia

29(34.52)

16(18.39)

0.023

Obesity (BMI > 30)

46(54.12)

43(49.43)

0.546

Coronary artery disease

$3(3.53)$

0

0.119

Congestive heart failure

$9(10.59)$

2(2.3)

0.031

Atrial fibrillation

8(9.41)

1(1.15)

0.017

Cerebrovascular accidents

11(12.94)

$2(2.30)$

0.009

Chronic Kidney Disease

11(12.94)

$5(5.75)$

0.121

Asthma

$5(5.88)$

3(3.45)

0.494

COPD

10(11.76)

$0(0.00)$

0.001

History of deep venous thrombosis

$4(4.71)$

2(2.30)

0.441

Cancer

10(11.76)

$2(2.30)$

0.017

Immunosuppression

12(14.12)

$2(2.30)$

0.005

Obstructive Sleep apnea

$3(3.53)$

3(3.45)

1

\section{Outcomes}

Hospitalization

63(74.12)

$58(66.67)$

0.319 


\section{Cureus}

\begin{tabular}{|llll|} 
ARDS & $19(22.35)$ & $14(16.09)$ & 0.336 \\
Sepsis & $20(23.53)$ & $21(24.14)$ & 1 \\
Shock & $11(12.94)$ & $8(9.20)$ & 0.474 \\
Mechanical Ventilation & $10(11.76)$ & $9(10.34)$ & 0.812 \\
Hepatic injury & $16(18.82)$ & $13(14.94)$ & 0.545 \\
Renal injury & $10(11.76)$ & $7(8.05)$ & 0.453 \\
Arrhythmia & $6(7.06)$ & $5(5.75)$ & 0.765 \\
Coagulopathy & $31(36.47)$ & $32(36.78)$ & 1 \\
Bleeding & $1(1.18)$ & $3(3.45)$ & 0.621 \\
Death & $13(15.29)$ & $5(5.75)$ & 0.048 \\
\hline
\end{tabular}

\section{TABLE 1: Baseline Characteristics and Outcomes According to Ethnicity}

Three comorbidities were found to significantly increase the risk of mortality among patients with COVID19: obesity, hypertension, and chronic obstructive pulmonary disease (Table 2). Hypertension and chronic obstructive pulmonary disease were significantly more common among non-Hispanics as compared to Hispanics (45 [52.9\%] versus 25 [28.7\%] and 10 [11.8\%] versus 0 [0\%], respectively). Other comorbidities that were more common among the non-Hispanic population include dyslipidemia, obesity, coronary artery disease, congestive heart failure, atrial fibrillation, cerebrovascular accidents, chronic kidney disease, asthma, cancer, and immunosuppression. However, the total sample size was not large enough to calculate the effect of these comorbidities on mortality.

\begin{tabular}{|c|c|c|c|}
\hline Predictor Variable & Odds Ratio & 95\% Confidence Interval & $p$-value \\
\hline Female & 0.58 & $0.09-3.66$ & 0.57 \\
\hline Age $>61$ years & 5.63 & $0.41-77.51$ & 0.2 \\
\hline Hispanic & 0.29 & $0.03-3.14$ & 0.31 \\
\hline Diabetes & 1.93 & $0.37-10.11$ & 0.44 \\
\hline Hypertension & 17.02 & $1.30-223.39$ & 0.03 \\
\hline Obesity (BMl> 30) & 10.55 & $1.07-104.45$ & 0.04 \\
\hline Chronic kidney disease & 1.61 & $0.21-12.40$ & 0.65 \\
\hline COPD & 15.45 & $1.33-178.98$ & 0.03 \\
\hline
\end{tabular}

\section{TABLE 2: Multivariable Logistic Regression Analysis of Mortality}

$\mathrm{BMI}=$ body mass index; $\mathrm{COPD}=$ chronic obstructive pulmonary disease.

Median household income was determined based on patient zip code. A total of 72 patients (41.9\%) lived in low-income neighborhoods; 100 (58.1\%) lived in middle- or upper-income neighborhoods. Hispanic individuals were significantly more likely than non-Hispanics to live in low-income zip codes (46 [52.9\%] versus 26 [30.6\%], respectively). Hispanics were also significantly more likely to be uninsured and less likely to have a primary care provider as compared to non-Hispanics (24 [27.6\%] versus 7 [8.2\%] and 37 [42.5\%] versus 52 [61.2\%], respectively).

Mortality was nearly threefold lower among Hispanics as compared to non-Hispanics (5 [5.8\%] versus 13 $[15.3 \%]$ ); the case fatality rate for Hispanics and non-Hispanics was $0.57 \%$ and $1.53 \%$, respectively. However, there was no significant difference in the rate of COVID-19-related in-hospital complications - including sepsis, shock, coagulopathy, and ARDS - between the two groups. The prothrombin time (PT) and D-dimer levels were significantly higher in non-survivors as compared to survivors; differences in PT and D-dimer were not significantly different between Hispanics and non-Hispanics $(p=0.06)$. 


\section{Discussion}

The relationship between ethnicity and COVID-19 represents a public health research priority. Existing data suggest a disproportionate burden of illness and death among minority communities [12]. However, the underlying causes of ethnic disparity in the setting of COVID-19 remain to be definitively established [7]. It has been widely hypothesized that socioeconomic factors - including work circumstances and living conditions - account for the increased incidence and mortality observed among minority communities. Indeed, ethnic minorities are markedly overrepresented in essential industries [13] and often live in multigenerational households [14,15]; practicing social distancing is therefore unrealistic if not impossible. As the renowned cardiologist and public health expert Clyde Yancy astutely observed in a recent editorial, "Being able to maintain social distancing... [is an issue] of privilege. In certain communities, these privileges are simply not accessible [9].”

Our findings demonstrate that Hispanics are disproportionately affected by COVID-19 in Northern Nevada. Hispanic individuals comprise only $25.7 \%$ of the population [16] but accounted for over half of the confirmed cases. Hispanic patients were significantly younger than their non-Hispanic counterparts, with a mean age of 46.0 versus 55.8 years, respectively. Interestingly, and perhaps consequently, Hispanics had fewer comorbidities and faced a threefold lower mortality rate than non-Hispanics. We propose that the increased prevalence of COVID-19 among young Hispanics reflects known sociodemographic patterns within the United States. Young Hispanic individuals frequently work in essential industries, such as food service and manufacturing [13], and are therefore unable to practice social distancing. Furthermore, as evidenced by our analysis of patient zip codes, Hispanics were more likely than non-Hispanics to reside in densely-populated, low-income neighborhoods. Increased risk of workplace exposure in the setting of limited social distancing inevitably creates a sociocultural environment favorable to the spread of disease.

It is conceivable that our data underestimate the prevalence of COVID-19 within the Hispanic community. Northern Nevada is home to a small but significant number of undocumented immigrants, and many Hispanic individuals who develop symptoms of COVID-19 may avoid seeking medical care. Indeed, fear of deportation has been established as a major barrier to health care access [17]. In the context of potential under-reporting, the disproportionate burden of disease among Hispanics is particularly striking.

Data on ethnic disparity in COVID-19 have demonstrated significantly increased mortality among Hispanics as compared to whites $[18,19]$; this finding is puzzling in light of the demographics of the United States, where the median age for Hispanics is 27 years versus 37 years for the general population. The age distribution in our study was consistent with national data: Hispanics diagnosed with COVID-19 were approximately one decade younger than their non-Hispanic counterparts. The case fatality rate of COVID19 increases with age [20], and therefore crude COVID-19-related deaths would be expected to be lower among Hispanics. Furthermore, morbidity and mortality data from the 2009

hemagglutinin type 1 and neuraminidase type 1 (H1N1) influenza pandemic - a similar global health crisis indicate that Hispanics were less susceptible to complications than non-Hispanics [21]. In light of the relative youth of the Hispanic populations and mortality patterns observed in the H1N1 influenza pandemic, why has excess COVID-19-related mortality been reported in numerous cities across the United States?

Interestingly, our findings are consistent with those from the neighboring state of California, where the case fatality rate was found to be slightly lower among Hispanics [22]. Studies on social determinants of health have shown that Hispanics face poverty and chronic illness at far greater rates than non-minorities [23] and would therefore be expected to experience excess mortality when compared to age-matched non-Hispanics. Indeed, this was established in a recent report by Gross et al., who concluded that the age-adjusted COVID19 mortality rate was nearly twice as high in Hispanics as compared to whites [24]. However, in contrast to our findings in Northern Nevada and those reported in California, crude mortality in many cities and states is higher among Hispanics than whites. For instance, in New York City, the crude death rate for Hispanics is 21.3 per 100,000 as compared to 15.7 per 100,000 in non-Hispanic whites and 9.1 per 100,000 in nonHispanic Asians [19]. Disparity of this magnitude warrants further investigation. We hypothesize that the comparatively low population density and disease burden of some states, such as California and Nevada, allows for greater access to health care than the urban epicenters; this may manifest as improved survival among minorities, who are conceivably less likely to delay treatment in the setting of equitable resource allocation.

It has also been postulated that susceptibility to infection may vary based on ethnic variation in allele distribution of the androgen receptor [25]. Biomolecular studies have demonstrated that the transmembrane protease, serine 2 (TMPRS22), which is regulated by androgen receptors, is required for SARS-CoV-2 infectivity [26]. Furthermore, epidemiologic data - including the present study - have established that COVID-19-related mortality is higher in men as compared to women [7,27]. It is therefore feasible that Hispanics express a variant of the androgen receptor that transcribes TMPRS22 at a higher rate than nonHispanics.

Community interventions targeting Hispanic communities have the potential to decrease the incidence of COVID-19 among this disproportionately affected and historically underserved group. Nearly one-fifth of Hispanic Americans speak only Spanish [28]; social distancing messaging should therefore be broadcast in 
both English and Spanish, particularly in Hispanic-predominant counties. Foreclosures and evictions should be suspended in order to prevent an increase in multi-individual and multi-generational households. Individuals in essential industries should be provided with resources that allow for telecommuting where possible. In an increasingly interconnected world, reducing ethnic health disparities improves the health not only of the Hispanic community, but of all communities nationwide.

\section{Conclusions}

Our single-center, retrospective observational study revealed that Hispanic individuals are disproportionately affected by COVID-19 in Northern Nevada. Hispanics tended to be younger and have fewer comorbidities than their non-Hispanic counterparts. Consequently, despite social determinants of health that would predict poorer outcomes - including low socioeconomic status, lack of insurance, and absence of a primary care provider - Hispanics experienced a significantly lower case fatality rate than nonHispanics. The underlying causes of these ethnic disparities warrant further investigation. However, in light of the marked overrepresentation of Hispanics in the COVID-19-positive population of Northern Nevada, targeted public health messaging has the potential to improve outcomes for the Hispanic community as well as the population at large.

\section{Additional Information \\ Disclosures}

Human subjects: Consent was obtained or waived by all participants in this study. Animal subjects: All authors have confirmed that this study did not involve animal subjects or tissue. Conflicts of interest: In compliance with the ICMJE uniform disclosure form, all authors declare the following: Payment/services info: All authors have declared that no financial support was received from any organization for the submitted work. Financial relationships: All authors have declared that they have no financial relationships at present or within the previous three years with any organizations that might have an interest in the submitted work. Other relationships: All authors have declared that there are no other relationships or activities that could appear to have influenced the submitted work.

\section{References}

1. Mann R, Perisetti A, Gajendran M, Gandhi Z, Umapathy C, Goyal H: Clinical characteristics, diagnosis, and treatment of major coronavirus outbreaks. Front Med. 2020, 7:581521. 10.3389/fmed.2020.581521

2. Perisetti A, Gajendran M, Boregowda U, Bansal P, Goyal H: COVID-19 and gastrointestinal endoscopies: current insights and emergent strategies. Dig Endosc. 2020, 32:715-722. 10.1111/den.13693

3. Worldometer - United States . (2021). Accessed: January 31, 2021: https://www.worldometers.info/coronavirus/country/us/.

4. Johnson KD, Harris C, Cain JK, Hummer C, Goyal H, Perisetti A: Pulmonary and extra-pulmonary clinical manifestations of COVID-19. Front Med. 2020, 7:526. 10.3389/fmed.2020.00526

5. Perisetti A, Gajendran M, Mann R, Elhanafi S, Goyal H: COVID-19 extrapulmonary illness - special gastrointestinal and hepatic considerations. Dis Mon. 2020, 66:101064. 10.1016/j.disamonth.2020.101064

6. Bibbins-Domingo K: This time must be different: disparities during the COVID-19 pandemic . Ann Intern Med. 2020, 173:233-234. 10.7326/M20-2247

7. Kopel J, Perisetti A, Roghani A, Aziz M, Gajendran M, Goyal H: Racial and gender-based differences in COVID-19. Front Public Health. 2020, 8:418. 10.3389/fpubh.2020.00418

8. Hooper MW, Nápoles AM, Pérez-Stable EJ: COVID-19 and racial/ethnic disparities. JAMA. 2020, 323:24662467. 10.1001/jama.2020.8598

9. Yancy CW: COVID-19 and African Americans. JAMA. 2020, 323:1891-1892. 10.1001/jama.2020.6548

10. Coward RT, Lee GR, Netzer JK, Cutler SJ, Danigelis NL: Racial differences in the household composition of elders by age, gender, and area of residence. Int J Aging Hum Dev. 1996, 42:205-227. 10.2190/WD8G-450QHC77-2K2G

11. Armstrong K, Ravenell KL, McMurphy S, Putt M: Racial/ethnic differences in physician distrust in the United States. Am J Public Health. 2007, 97:1283-1289. 10.2105/AJPH.2005.080762

12. Garg S, Kim L, Whitaker M, et al.: Hospitalization rates and characteristics of patients hospitalized with laboratory-confirmed coronavirus disease 2019 - COVID-NET, 14 states, March 1-30, 2020. MMWR Morb Mortal Wkly Rep. 2020, 69:458-464. 10.15585/mmwr.mm6915e3

13. Byars-Winston A, Fouad N, Wen Y: Race/ethnicity and sex in U.S. occupations, 1970-2010: implications for research, practice, and policy. J Vocat Behav. 2015, 87:54-70. 10.1016/j.jvb.2014.12.003

14. Berchick ER, Barnett JC, Upton RD: Health Insurance Coverage in the United States: 2018. Current Population Reports. US Government Printing Office, Washington DC; 2019.

15. Census.gov: multigenerational households. (2013). Accessed: May 22, 2020: https://www.census.gov/content/dam/Census/library/working-papers/2013/acs/lofquist-01.pdf.

16. US Census Bureau quick facts: Reno, Nevada. (2020). Accessed: January 31, 2021: http://www.census.gov/quickfacts/renocitynevada.

17. Hacker K, Anies ME, Folb BL, Zallman L: Barriers to health care for undocumented immigrants: a literature review. Risk Manag Healthc Policy. 2015, 8:175-183. 10.2147/RMHP.S70173

18. Chicago Department of Public Health: latest data . (2020). Accessed: May 22, 2020: https://www.chicago.gov/city/en/sites/covid-19/home/latest-data.html.

19. NYC Health: COVID-19 data. (2021). Accessed: January 31, 2021 https://www1.nyc.gov/site/doh/covid/covid-19-data.page.

20. Mahase E: COVID-19: death rate is $0.66 \%$ and increases with age, study estimates . BMJ. 2020, 369:1327. 


\section{Cureus}

10.1136/bmj.m1327

21. Quinn SC, Kumar S, Freimuth VS, Musa D, Casteneda-Angarita N, Kidwell K: Racial disparities in exposure, susceptibility, and access to health care in the US H1N1 influenza pandemic. Am J Public Health. 2011, 101:285-293. 10.2105/AJPH.2009.188029

22. California Department of Public Health: COVID-19 race and ethnicity data . (2021). Accessed: January 31, 2021: https://www.cdph.ca.gov/Programs/CID/DCDC/Pages/COVID-19/Race-Ethnicity.aspx.

23. Velasco-Mondragon E, Jimenez A, Palladino-Davis AG, Davis D, Escamilla-Cejudo JA: Hispanic health in the USA: a scoping review of the literature. Public Health Rev. 2016, 37:31. 10.1186/s40985-016-0043-2

24. Gross CP, Essien UR, Pasha S, Gross JR, Wang SY, Nunez-Smith M: Racial and ethnic disparities in population level COVID-19 mortality. J Gen Intern Med. 2020, 35:3097-3099. 10.1007/s11606-020-06081-w

25. McCoy J, Wambier CG, Vano-Galvan S, et al.: Racial variations in COVID-19 deaths may be due to androgen receptor genetic variants associated with prostate cancer and androgenetic alopecia. Are anti-androgens a potential treatment for COVID-19?. J Cosmet Dermatol. 2020, 19:1542-1543. 10.1111/jocd.13455

26. Hoffmann M, Kleine-Weber H, Schroeder S, et al.: SARS-CoV-2 cell entry depends on ACE2 and TMPRSS2 and is blocked by a clinically proven protease inhibitor. Cell. 2020, 181:271-280.E8.

10.1016/j.cell.2020.02.052

27. Meng Y, Wu P, Lu W, et al.: Sex-specific clinical characteristics and prognosis of coronavirus disease-19 infection in Wuhan, China: a retrospective study of 168 severe patients. PLoS Pathog. 2020, 16:e1008520. 10.1371/journal.ppat.1008520

28. Rumbaut RG, Massey DS: Immigration and language diversity in the United States . Daedalus. 2014, 142:141-154. 10.1162/daed a 00224 